\title{
Intelligent Collection of Detection Data and Automatic Generation of Report Based on LabVIEW in ABS Adhesion Coefficient Utilization Test
}

\author{
Yucheng DU ${ }^{\mathrm{a}, 1}$ and Hao $\mathrm{LI}^{\mathrm{a}}$ \\ ${ }^{a}$ Research Institute of Highway Ministry of Transport, Beijing 100088, China
}

\begin{abstract}
The automobile anti-lock braking system (ABS) is a hot spot in the current research and development of automobiles. The country also has corresponding standards for its performance requirements and test methods. Among them, the utilization rate of the adhesion coefficient is one of its important performance evaluation indicators. In the adhesion coefficient utilization test, the post-processing workload of the data is large, and the authenticity of the data is crucial to the test results. In order to overcome the shortcomings of manual reporting, which takes a long time and is prone to human errors. Based on the LabVIEW platform, this paper constructs a method for automatic generation of test reports, and designs a comprehensive program for automatic report generation. Experimental shows that the use of integrated automation software to issue an adhesion coefficient utilization test report can reduce data post-processing work and greatly improve the efficiency and accuracy of the report.
\end{abstract}

Keywords. Anti-lock braking system(ABS), adhesion coefficient utilization test, test report, LabVIEW, automatic generation

\section{Introduction}

With the rapid development of society and the continuous increase in demand, the testing institutions will need to deal with more and more test data. The issuance of the test report must strictly follow the requirements of the national standard, and the authenticity, standardization and traceability of the test data must be guaranteed. At present, the post-processing process of the adhesion coefficient utilization test effectively collects the required data from a large amount of test data, and then subjectively judges whether it meets the requirements. The calculation and analysis results need to be output in the form of a specific report template, and then the user can achieve the test results. But it will solve many problems, such as a wide range of testing instruments and equipment, inconsistent testing data formats, and difficult information interaction between equipment; low level of automation in testing data processing, and manual judgment of test results, which is time-consuming; test reports rely on manual entry, repeated filling of report standard data is a heavy workload, at the

\footnotetext{
${ }^{1}$ Yucheng Du, Research Institute of Highway Ministry of Transport, Beijing 100088, China; E-mail: 15001057252@163.com.
} 
same time, manual copying and modification of data is easy to cause work errors, which inevitably reduces the efficiency and accuracy of the detection work.

Therefore, aiming at the current situation of most automobile testing institutions, this paper studies the technologies such as original data reading and report generation based on LabVIEW platform, and successfully applies the system to the test project of adhesion coefficient utilization of automobile anti lock braking system performance, in order to improve the detection efficiency and automation level, and better avoid the shortage of manual output report, It has important engineering practical significance and practical research value.

\section{Adhesion Coefficient Utilization Test Method}

The automobile anti-lock braking system is a safety device that prevents the wheels from locking during the braking process, improves the directional stability and steering ability, and shortens the braking distance [1]. GB13594-2003 and GB 21670-2008 stipulate the performance requirements and test methods of anti-lock braking systems, in which the utilization factor of the adhesion coefficient and additional test are the main evaluation indicators [2]. Through the wheel speed sensors installed on the wheels, attachment test is to observe whether the car's wheels are locked on the road with different adhesion coefficients, This test process is simple and the test results are intuitive. However, the test process of the adhesion coefficient utilization rate is complicated [3-4], the test results are poor in repeatability, the data post-processing workload is large, and the test report generation cycle is long.

\subsection{Adhesion Coefficient Utilization Test Calculation Process}

The adhesion coefficient utilization rate is defined as the quotient of the maximum braking strength and the adhesion coefficient when the anti-lock braking system is working:

$$
\varepsilon=\frac{Z_{A L}}{k_{M}}
$$

Measure the maximum braking strength. The initial braking speed is $55 \mathrm{~km} / \mathrm{h}$. When the full cycle of the anti-lock brake system, multiple tests are carried out by increasing the pipeline pressure successively to determine the maximum braking strength of the vehicle. During each test, keep the pedal force constant, measure the time when the speed drops from $45 \mathrm{~km} / \mathrm{h}$ to $15 \mathrm{~km} / \mathrm{h}$, start from the minimum measured value of $\mathrm{t} t_{\min }$, and choose 3 values between $t_{\min }$ and $1.05 t_{\min }$, calculate its arithmetic mean $t_{m}$, if the above 3 values cannot be obtained, the shortest time $t_{\min }$ can be used. Calculate the maximum braking strength.

$$
Z_{A L}=\frac{0.849}{t_{m}}
$$

The adhesion coefficient is determined by the quotient of the maximum braking force divided by the corresponding dynamic axle load of the braked axle under the 
premise of no wheel lock. For example, when determining the adhesion coefficient of a front-wheel drive two-axle vehicle, only a single axle of the test vehicle is braked, and the initial braking speed is $50 \mathrm{~km} / \mathrm{h}$. At this time, the anti-lock braking system should be disengaged or not working. According to the operation method and time selection principle when determining the maximum braking intensity, the $t_{m}$ from $40 \mathrm{~km} / \mathrm{h}$ to $20 \mathrm{~km} / \mathrm{h}$ is measured, and the wheels are not allowed to lock during $t_{m}$. Then it is calculated by the following formula:

$$
\begin{gathered}
k_{f}=\frac{Z_{m} \times P \times g-0.01 F_{2}}{F_{1}+\frac{h}{E} \times Z_{m} \times P \times g} \\
k_{r}=\frac{Z_{m} \times P \times g-0.015 F_{1}}{F_{2}-\frac{h}{E} \times Z_{m} \times P \times g} \\
F_{f d y n}=F_{f}+\frac{h}{E} \times Z_{A L} \times P \times g \\
F_{r d y n}=F_{r}-\frac{h}{E} \times Z_{A L} \times P \times g \\
k_{M}=\frac{k_{f} \times F_{f d y n}+k_{r} \times F_{r d y n}}{P \times g}
\end{gathered}
$$

Among them: $Z_{\mathrm{m}}$ is the average braking strength, $k_{\mathrm{f}}$ or $k_{\mathrm{r}}$ is the adhesion coefficient of a front or rear axle, $F_{i}$ is the normal static reaction force of the road to the i axis, $P$ is the static weight vehicle, $h$ is the height center gravity, and $E$ is the distance between axles 1-2.

\subsection{Test Data Collection}

On the ABS performance test road of Guangde automobile inspection field, the adhesion coefficient utilization test adopts the empty and full load states to test on the high adhesion coefficient road and the low adhesion coefficient road. The ABS system of the test vehicle is dual-circuit hydraulic drive, diagonally arranged, and four-channel independent control type. The following are the test results of a domestic light passenger car with Type 1 ABS. The test car technical parameters are shown in table 1. During the test, the test data in EXCEL format is generated by the upper computer, data collection in table 2 and table 3.

Table 1. Parameters of the test car.

\begin{tabular}{llllll}
\hline & $\begin{array}{l}\text { Static weight } \\
1 \text { st axle } \\
(\mathrm{kg})\end{array}$ & $\begin{array}{l}\text { Static weight } \\
\text { 2nd axle } \\
(\mathrm{kg})\end{array}$ & $\begin{array}{l}\text { Static weight } \\
\text { vehicle } \\
(\mathrm{kg})\end{array}$ & $\begin{array}{l}\text { Height center } \\
\text { gravity } \\
(\mathrm{mm})\end{array}$ & $\begin{array}{l}\text { Distance } \\
\text { between axles } \\
1-2(\mathrm{~mm})\end{array}$ \\
\hline Unladen & 2466 & 5060 & 7526 & 945 & 3600 \\
\hline Laden & 6823 & 11426 & 18249 & 1525 & 3600 \\
\hline
\end{tabular}


Table 2. Low adhesion coefficient utilization test.

\begin{tabular}{lllll}
\hline Load & Brake test & $\mathrm{t}_{1}$ & $\mathrm{t}_{2}$ & $\mathrm{t}_{3}$ \\
\hline \multirow{5}{*}{ Unladen } & $\begin{array}{l}\text { All axles break } \\
\text { (ABS system on) }\end{array}$ & 8.880 & 8.800 & 8.590 \\
\cline { 2 - 5 } & $\begin{array}{l}\text { 1st axle break } \\
\text { (ABS system failure) }\end{array}$ & 7.000 & 6.830 & 6.940 \\
\cline { 2 - 5 } & $\begin{array}{l}\text { 2nd axle break } \\
\text { (ABS system failure) }\end{array}$ & 15.520 & 15.330 & 15.220 \\
\hline \multirow{3}{*}{ Laden } & $\begin{array}{l}\text { All axles break } \\
\text { (ABS system on) }\end{array}$ & 9.550 & 9.560 & 9.510 \\
\cline { 2 - 4 } & $\begin{array}{l}\text { 1st axle break } \\
\text { (ABS system failure) }\end{array}$ & 13.210 & 12.830 & 13.370 \\
\cline { 2 - 4 } & $\begin{array}{l}\text { 2nd axle break } \\
\text { (ABS system failure) }\end{array}$ & 10.300 & 10.350 & 9.870 \\
\hline
\end{tabular}

Table 3. High adhesion coefficient utilization test.

\begin{tabular}{lllll}
\hline Load & Brake test & $\mathrm{t}_{1}$ & $\mathrm{t}_{2}$ & $\mathrm{t}_{3}$ \\
\hline \multirow{3}{*}{ Unladen } & $\begin{array}{l}\text { All axles break } \\
\text { (ABS system on) }\end{array}$ & 1.180 & 1.220 & 1.200 \\
\cline { 2 - 5 } & $\begin{array}{l}\text { 1st axle break } \\
\text { (ABS system failure) }\end{array}$ & 1.000 & 1.030 & 0.940 \\
\cline { 2 - 5 } & $\begin{array}{l}\text { 2nd axle break } \\
\text { (ABS system failure) }\end{array}$ & 3.360 & 3.360 & 3.360 \\
\hline \multirow{2}{*}{ Laden } & $\begin{array}{l}\text { All axles break } \\
\text { (ABS system on) }\end{array}$ & 1.410 & 1.400 & 1.400 \\
\cline { 2 - 5 } & $\begin{array}{l}\text { 1st axle break } \\
\text { (ABS system failure) }\end{array}$ & 1.730 & 1.770 & 1.690 \\
\cline { 2 - 5 } & $\begin{array}{l}\text { 2nd axle break } \\
\text { (ABS system failure) }\end{array}$ & 1.920 & 1.920 & 1.860 \\
\hline
\end{tabular}

\section{System Key Technology Realization}

\subsection{LabVIEW Detection Data Reading Technology Based on ActiveX Control}

The software environment is based on LabVIEW2020 and MS EXCEL 2016. The LabVIEW detection data reading technology based on ActiveX controls mainly uses six functions including variant-to-data conversion, open automation, close reference [5], attribute node, call node, and conversion to variant. Through the above functions, any required ActiveX functions can be realized in LabVIEW, and these functions as an abstraction level greatly simplify programming [6-7]. Read the data in the EXCEL table in LabVIEW, and the design steps of the ActiveX software block diagram are as follows:

1) Add an automation reference handle on the front panel, right-click on the block diagram, click the reference handle in the control, select the automation reference handle, select "ActiveX Class" from the menu, and select "Microsoft EXCEL 12.0 Object" in the type library Library Version 1.6", select "_Application" in the object option box to complete the creation of an EXCEL reference handle.

2) Add an attribute node of "_Application", set its attribute to "Workbooks", and connect it to the upper level attribute node. 
3) Add a call node and connect it to the "Workbooks" of the upper-level attribute node. the name of the call node will also be changed to "Workbooks". Select the "Open" method in the tab. Create a file path input control on the front panel, and use the path-to-string conversion function on the block diagram to convert its type to a string, and finally connect it to the "Filename" property under the "Open" method to open the EXCEL file route of.

4) Add the reference node of "Worksheets", choose to call its "Item" method, and create a string constant "Sheet1" which is converted into a variant function and connected to its "Index" parameter to index the worksheet in the file. Add another automation reference handle, select "_Worksheet" in the object option box, and connect to the "EXCEL._Worksheet" automation reference handle.

5) Add the attribute node of "_Worksheet", select the attribute of "UsedRange", add the call node of "Range", and "Value" outputs the data information in the cell range. Add a variant to the data conversion function, input a string constant array, and convert the data information into a two-dimensional array form.

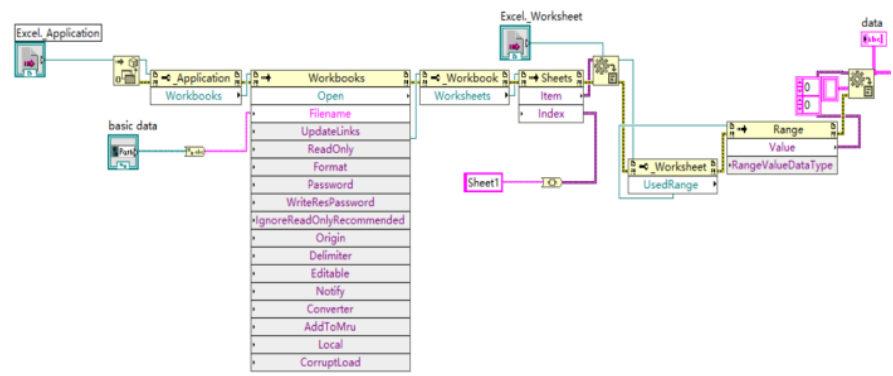

Figure 1. Block diagram of the procedure for reading file data.

The tested data is transferred to the system folder through the RS232 serial port, and the detection data reading module uses Active X control to open the EXCEL file method. Figure 1 is the block diagram of the function of reading the EXCEL file data. Create a file path input control and add the detection data file [8]. You can open the file at the location and read the data required by the file directory to open the EXCEL file.

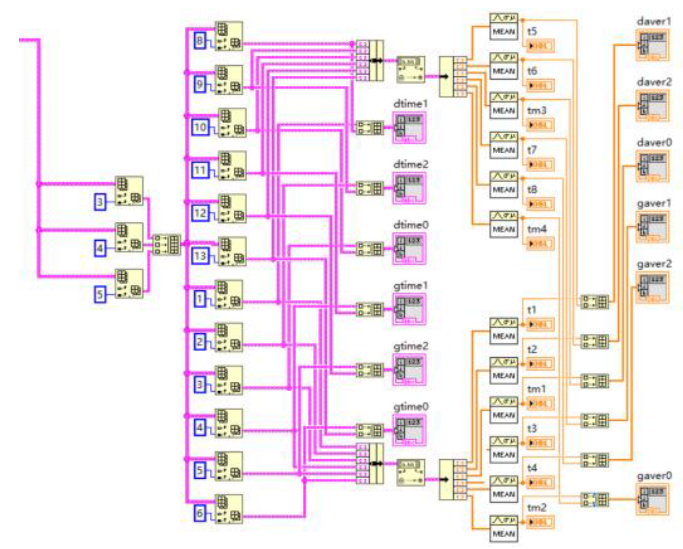

Figure 2. Block diagram of testing data formatting and classification process. 
Figure 2 is a block diagram of the format of the detection data. The detection data read from EXCEL is in string format, and the length of the output data of the automation equipment does not meet the requirements, and cannot be used for comparison and entry with standard data [9]. Use LabVIEW bundled functions to pack each detection data into a cluster format to facilitate data batch processing. Convert the collected data into double-precision numbers using the fractional string to numeric function. Unbind the clusters and convert the rate of change in the report to percentage format data; bind the clusters again, convert the cluster data into a two-dimensional array through a function and retain 3 decimal places of precision.

\subsection{EXCEL report generation technology based on LabVIEW}

Base on Excel report generation technology mainly uses several basic functions such as creating new reports, setting report fonts, adding report text, adding tables to reports, disposing of reports, and saving reports to files [10]. The process of creating a test report is as follows:

1) Create a new Excel document of report template, enter the preset template information in the document, and add Bookmarks where the information needs to be entered. The LabVIEW report generation function will write based on the bookmark position.

2) Place a new report function in the block diagram, select Excel as the report generation option, and right-click to create an "input control" at the connection end of the template to generate a template path input control. When running, the control will open the corresponding report template file for reading.

3) Place the report text function on the block diagram, and connect its report input terminal to the created output terminal of the report font function. Right-click on the text terminal to add a string constant or input control as a text input parameter; rightclick on the MS Office parameter terminal to create a constant, and the new cluster will contain the position and name of the Excel row and column where the text is inserted.

4) Place the add form to the report function in the block diagram, connect its report input terminal with the report input terminal of the previous step, right click to add a two-dimensional array constant or input control to the text data terminal; right click to add it to the MS Office parameter terminal Excel bookmark location. Place the save report to file function on the block diagram, add a path input control to the report file path terminal, and specify the save location of the generated report. Figure 3 is a block diagram of the Excel report generation program based on LabVIEW.

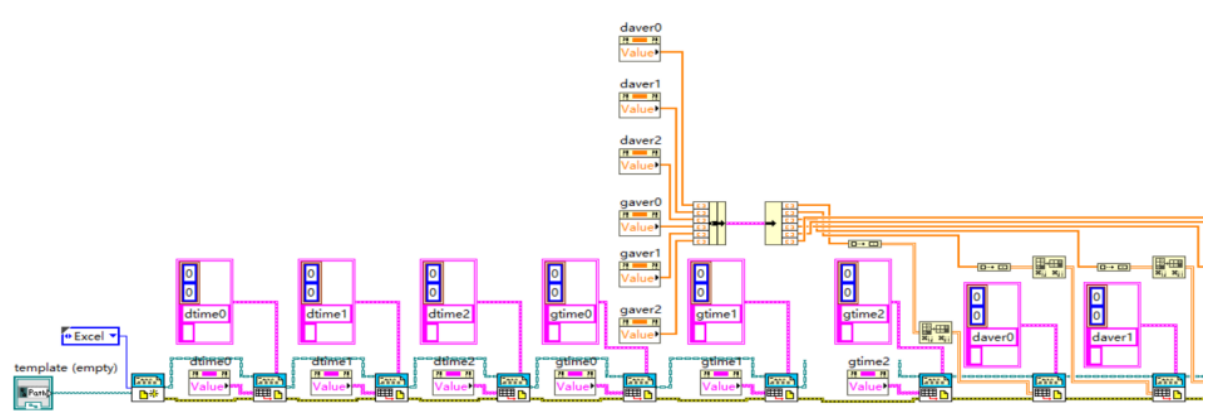

Figure 3. Block diagram of Excel report generation based on LabVIEW. 

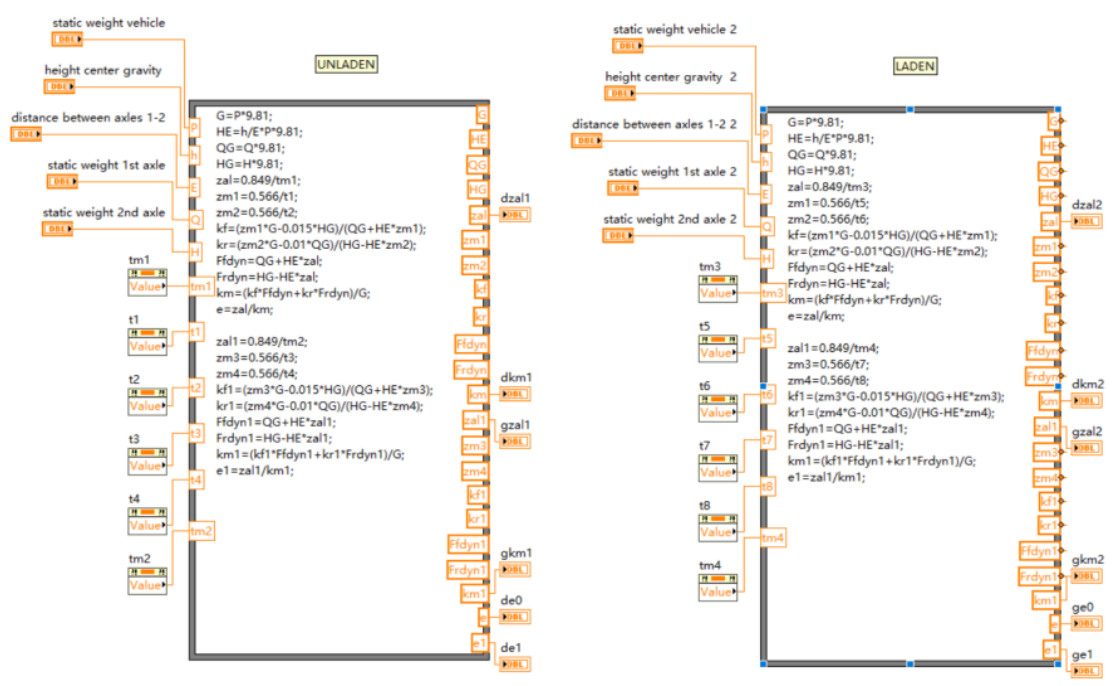

Figure 4. Block diagram of formula calculation procedure.

The most important information of the test report is undoubtedly the final test result. By comparing whether the test data is within the allowable range of the standard data, it is judged whether a product parameter meets the standard requirements. First, perform calculation processing on the formatted data, use the formula node control in the number function to manually enter the basic parameters of the car model with a numeric input control, and assign the formatted data by creating a node value, and output the standard The required value, figure 4 is the block diagram of the formula calculation procedure. The program is in a "For" loop structure, the program loops through each item in the detection data array, the value comparison function judges, and the corresponding bookmark mark position in the test report is compared to output each test result. If it meets the requirements of the standard, output "P" to the test report, otherwise output "F". Figure 5 is the block diagram of the detection data judgment process.

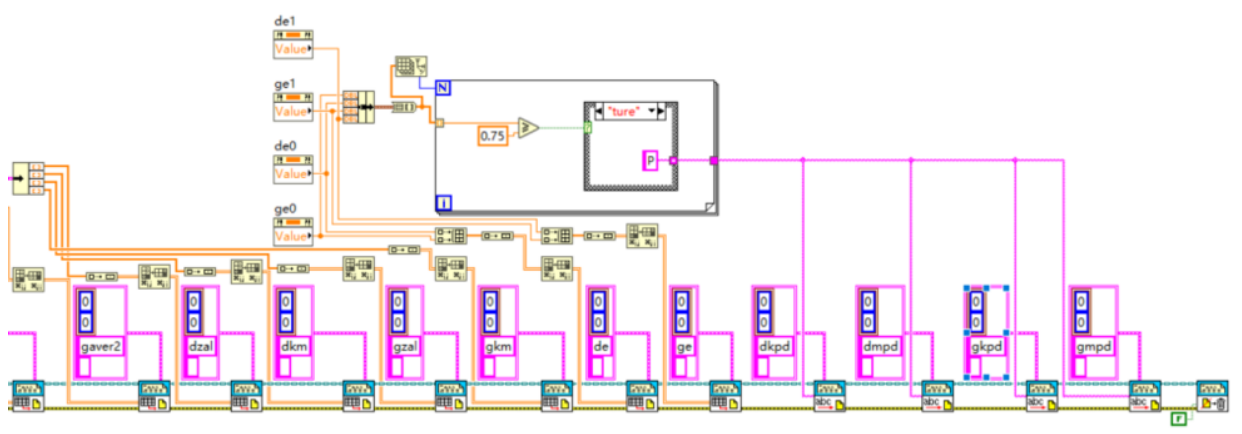

Figure 5. Block diagram of detection data judgment process. 


\section{Application Effect Analysis}

Figure 6 shows the adhesion coefficient utilization test report automatically generated by the system. After an automobile testing agency actually tried this system, in the process of issuing a test report, the original manual entry method took about 20 minutes to complete the test data collection, data processing, result evaluation, data entry, report printing, etc.; after using this system, the entire The process of intelligent collection of adhesion coefficient utilization detection data and report generation is automatically processed by the software. Standard requirements, inspection results, and individual evaluations are automatically entered by the system. The generated inspection report is completely consistent with the original manual input report. It only takes about 1 minute to complete all the work. Compared with the original method, it saves about $95 \%$ of the time and cost, and greatly improves the efficiency of data collection and report generation. At the same time, it eliminates the possibility of operators manually inputting wrong data, and improves the reliability of detection data. In the testing industry, it has very good promotion value.

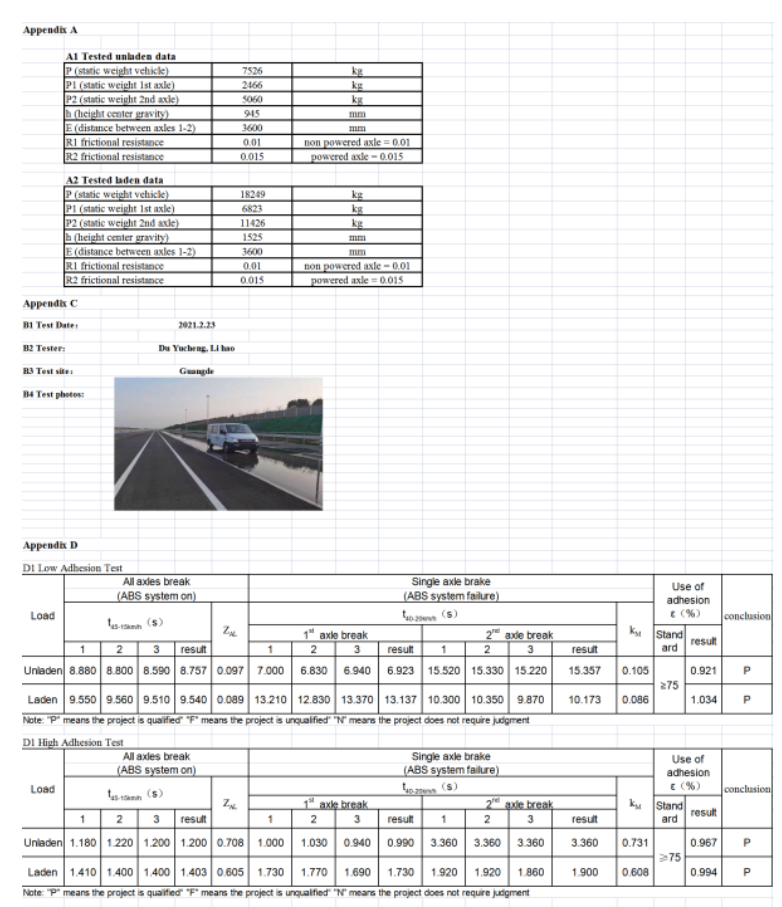

Figure 6. Automatically generated adhesion coefficient utilization test report.

\section{Conclusion}

Aiming at the current deficiencies in data collection and report generation in various testing equipment, this paper proposes the idea of intelligent collection and report generation of test data based on LabVIEW, and discusses in detail the key points of LabVIEW test data reading based on ActiveX and Excel report generation of 
LabVIEW. Technology, and successfully applied the system to the adhesion coefficient utilization test calculation of the automobile anti-lock brake system test, researched and used ActiveX control to detect data reading technology, Excel report generation and other key technologies to realize intelligent data collection, report generation, and results judgment and other functions. Through the application experiment, verify the feasibility of each module function, improve the detection efficiency and automation level.

\section{References}

[1] Sun JT,Liu L,Cui Y. Influence of antilock brake system on braking distance. Automobile Parts.2020 Dec; (12): 73-75.

[2] Xia XJ, Zhang XL, Feng QF. Design and test of integrated performance test system for pneumatic antilock braking system in commercial vehicles. Automotive Engineering.2020 Feb; 42(2): 215-221.

[3] Wu J. Research on road testing method of automobile anti-lock braking system. Heavy Truck. 2020 Feb; (2): 7-9.

[4] Xu XG, Li EK. Research on ABS test method of n2 light truck. Automation \& Instrumentation. $2016 \mathrm{O}$ ct; (10): 45-49.

[5] Lu X, Bao XM, Rao ZM.Data Recording and database access technology based on LabView. Journal of Hubei Minzu University (Natural Science Edition). 2019 Jun; 37(2): 201-205.

[6] Cao YB, Zhou ZQ, WU Kai. Research on Word format test report Based on ActiveX technology in Labview. China lntegrated Circult. 2016 Jun; 25(6): 69-72.

[7] Liu X,Wang J, Ju GG. Design and implementation of test report generation system based on LabView and SQL Server. Ship Electronic Engineering. 2018 May; 38(5): 93-97.

[8] Zhang WF Zeng JH. Automatic generation method of original record and calibration certificate based on LabVIEW. Metrology \& Measurement Technique. 2018 Sep; 45(9): 95-97.

[9] Huang X, Meng J, Tang J. LabVIEW-based report generation technique in the Warship's multichannel test system marine electric. 2015 Jun; 35(6): 15-23.

[10] Yang JT, Li T, Sun QH. Complex word report customization based on ActiveX in LabVIEW. Journal Of Wuyi University (Natural Science Edition). 2016 Feb; 30(1): 69-72. 